IN THIS ISSUE

\section{Assessing ARID1B spectrum patients}

https://doi.org/10.1038/s41436-018-0330-z

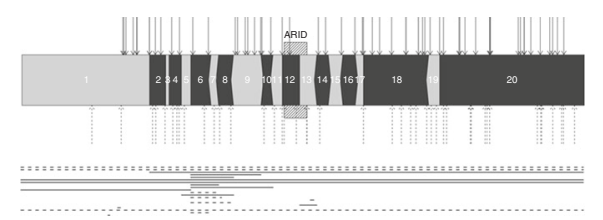

Pathogenic variants in the gene $A R I D 1 B$ can lead to Coffin Siris syndrome (CSS), a rare condition marked by neurodevelopmental abnormalities including intellectual disability, minor skeletal anomalies, coarse facial features, and other minor facial dysmorphic features as well other highly variable features. However, pathogenic variants in $A R I D 1 B$ can also lead to nonsyndromic intellectual disability (ID). It is unclear whether issues including ascertainment bias and phenotype underreporting bias have impacted the previous understanding of the CSS-versus-ID phenotypes associated with ARID1B pathogenic variants. In this issue, van der Sluijs et al. report a comparison of genotypic and phenotypic differences between Coffin Siris syndrome patients and ID patients with pathogenic $A R I D 1 B$ variants. The researchers developed an online survey to which they and other clinicians submitted data from 143 patients with pathogenic $A R I D 1 B$ variants, including 79 patients previously diagnosed with CSS. The online survey was carefully designed to allowed researchers to discriminate between the verified absence of a feature in a patient and data clinicians failed to assess or submit. This is important since, as the authors point out, instruments such as the Human Phenotype Ontology may underestimate gene-related features. The survey revealed that, as expected, CSS diagnosed patients with pathogenic ARID1B variants displayed minor dysmorphic features associated with the syndrome more frequently than ID patients who also harbor pathogenic $A R I D 1 B$ variants, including thick eyebrows, long eyelashes, thick alae nasi, a long and/or broad philtrum, small nails, and small or absent fifth distal phalanx and hypertrichosis $(p<0.0001-0.001)$. The researchers did not find any other significant differences between the cohorts. All pathogenic variants in $A R I D 1 B$ in both cohorts were truncating. The authors conclude that only subtle differences exist between CSSdiagnosed patients with pathogenic $A R I D 1 B$ variants and ID patients with the same genetic underpinning. They go on to suggest that $A R I D 1 B$ disorders represent a phenotypic spectrum, with the classic patient typically displaying several features including ID, feeding difficulties, laryngomalacia, speech delay, motor delay, hypertrichosis, and cryptorchidism while others may lack many of the dysmorphic features and other anomalies. The recommendation is made that patients with $A R I D 1 B$ pathogenic variants and labeled as ARID1B-ID should nevertheless be evaluated for complications of the full CSS phenotypic spectrum. Survey design to accurately capture true absence of features as opposed to lack of ascertainment is also discussed. - V. L. Dengler, News Editor

\section{Genomic mosaicism affects Rett syndrome pathogenicity}

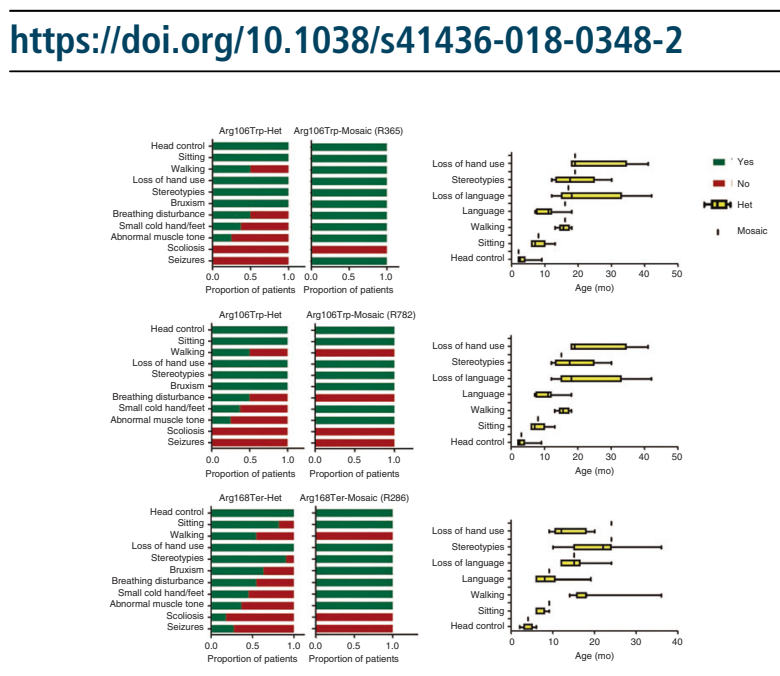

Children with Rett syndrome, a genetic neurodevelopmental disorder, exhibit normal early growth and development followed by a slowing of development, loss of motor skills, seizures, and intellectual disability. Pathogenic variants in methyl-CpG-binding protein 2 (MECP2) cause 95\% of classical Rett syndrome cases. Variants in cyclin-dependent kinase-like 5 (CDKL5) and Forkhead box protein G1 (FOXG1) lead to similar, overlapping phenotypes. However, some patients with Rett syndrome or Rett-like phenotypes do not harbor pathogenic variants in $M E C P 2, C D K L 5$, or FOXG1. In this issue, Zhang et al. report that somatic MECP2 mosaicism plays a direct role in the syndrome's pathogenicity. The researchers screened 147 Rett and Rett-like syndrome patients who did not show pathogenic variants in $M E C P 2$, CDKL5, or FOXG1 for somatic mosaicism using nextgeneration sequencing and analysis by MosaicHunter. Potential mosaic variants were validated by microdroplet digital PCR. Five (1.1\%) of the 471 total patients included in the study exhibited somatic MECP2 mosaicism. Four of the five somatic MECP2 mosaic patients had Rett syndrome and one had Rett-like syndrome; three were males $(17 \%, 3 / 18)$ and two were females $(0.4 \%, 2 / 453)$. Mutant allelic fractions varied from 6.5 to $38 \%$. Three of the five patients with somatic MECP2 mosaicism showed mosaicism at MECP2-Arg106. The finding suggests that this residue may be a mutational hotspot. Surprisingly, patients with MECP2 mosaicism exhibited comparable or even more severe Rett syndrome symptoms than patients with variants at the same genomic positions. The researchers also detected germline MECP2 mosaicism in five fathers $(24 \%, 5 / 21)$. The discovery indicates that paternal germline $M E C P 2$ mosaicism potentially underlies the paternal origin bias of $M E C P 2$ variants. The authors conclude that clinicians should consider MECP2 mosaicism when evaluating patients with Rett-like phenotypes, especially males. - V. L. Dengler, News Editor 